\title{
Inhalt des 15. Bandes
}

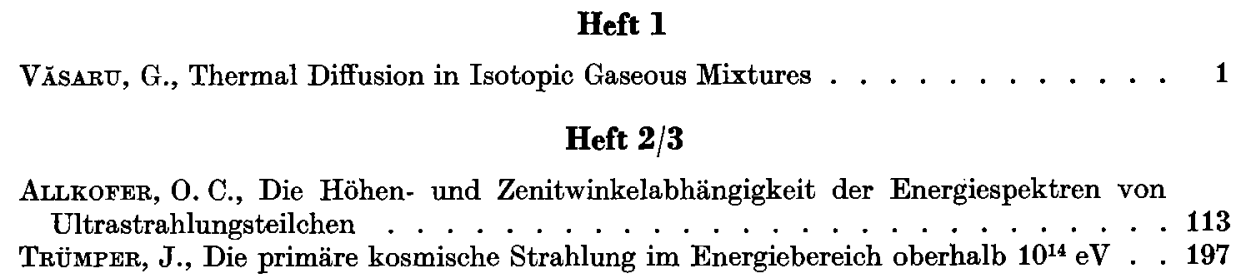

\section{Heft 4}

SExL, R. U., Theorie of Gravitation . . . . . . . . . . . . . . . . . . . . . . . 269

WastPfand, K., Allgemeine Relativitätstheorie und Minkowskische Vergleichsmetrik 309

\section{Heft $5 / 6$}

OTTER, G., The K-Meson-Proton Interaction at Low Energies . . . . . . . . . . . 333

MaRTIN, B. R., An Introduction of Some Recent Work in Weak Interactions . . . . . 357

Schmidt, W., und G. Schwiderskr, Application of Helicity-Formalism in Pion-Photo-

production . . . . . . . . . . . . . . . . . . . 393

\section{Heft 7}

Barashenkov, V. S., und V. M. Maltsev, Inelastic Interactions of Particles at High

Energies II. (Energy and angular distributions of secondary particles) . . . . . . 435

\section{Heft 8}

Lehar, F., and P. Winternitz, Polarized Proton Targets and Low-Energy Nucleon-

Nucleon Experiments . . . . . . . . . . . . . . . . . . . . . . . . . . . . 495

GrLson, J. G., Independent Electron Positron Pair. Production Events and Energy Loss 537

\section{Heft 9}

Brandt, S., Neuere Ergebnisse zur Pion-Proton-Streuung bei hohen Energien . . . . 551

\section{Heft 10}

Hayashi, K., Hirayama, M., Muta, T., Seto, N., and T. Shrrafuji, Compositeness Criteria of Particles in Quantum Field Theory and S-Matrix Theory . . . . . . . 625

\section{Heft 11}

Axs, S., On the Connections Between the Non-Linearity of Equations of Motion in Quantum Theory, Asymptotic Behavior and Renormalization . . . . . . . . . . . . 661

LOPUSZanSKI, J. T., On Goldstone's Theorem . . . . . . . . . . . . . . . . . . . 681

\section{Heft 12}

ANSARI, S. M. R., I. An Operator Method for the Addition of Two Angular Moments by Symmetrizing the Product on $n$ Spin Functions . . . . . . . . . . . . . . 707

ANSARI, S. M. R., II. Quasi-binomial Representations of Clebsch-Gordan Coefficients 729

StIller, H., Lucke, O., und F. C. WAGNer, Neuere Ergebnisse aus der Geophysik II. Physik der Erdkruste und des oberen Erdmantels Teil 2. Methoden und Ergebnisse der geophysikalischen Festkörperforschung bei der Untersuchung der physikalisch-chemischen Eigenschaften des Erdmantels 
Herausgeber: Prof. Dr. Frank Kaschluhn, Prof. Dr. Artur Lösche, Prof. Dr. Rudolf Ritschl und Prof. Dr. Robert Rompe; Manuskripte sind zu richten an die Schriftleitung: Dr. Lutz Rothkirch, II. Physikalisches Institut der Humboldt-Universität Berlin, 104 Berlin, Hessische Str. 2. Verlag: Akademie-Verlag GmbH, 108 Berlin, Leipziger Str. 3/4, Fernruf: 220441, Telex-Nr. 011 2020, Postscheckkonto: Berlin 35021. Die Zeitschrift „Fortschritte der Physik" erscheint monatlich; Bezugspreis eines Heftes 8, - (Sonderpreis für die DDR 6,- M). Bestellnummer dieses Heftes: 1027/15. - Satz und Druck: VEB Druckhaus „Maxim Gorki“, 74 Altenburg, Bez. Leipzig, Carl-von-Ossietzky-Str. 30-31. - Veröffentlicht unter der Lizenznummer 1324 des Presseamtes beim Vorsitzenden des Ministerrates der Deutschen Demokratisehen Republik. 


\section{FORTSCHRITTE DER}

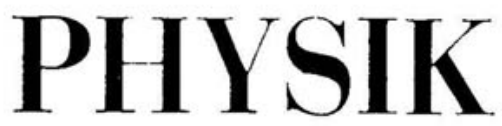

HERAUSGEGEBEN IM AUFTRAGE. DER PHYSIKALISCHEN GESELLSCHAFT IN DER

DEUTSCHEN DEMOKRATISCHEN REPUBLIK

VON F. KASCHLUHN, A. LÖSCHE, R. RITSCHL UND R. ROMPE

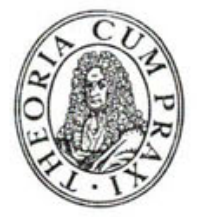

A K A D E M I E - V E R L A G B E R L I N 


\section{Ausgleichsrechnung mit Anwendungen in der Physik}

unter besonderer Berücksichtigung der Meßtechnik

(Technisch-physikalische Monographien, Band 20)

Von Dr. E. Hultzsch, Jena

1966. 136 Seiten mit 9 Bildern und 8 Tabellen. Format: $147 \times 215 \mathrm{~mm}$

Ganzleinen 19,50 MDN

Der Verfasser behandelt das Grundlegende der Theorie der Ausgleichsrechnung in einer möglichst einfachen Darstellung, während er das Schwergewicht auf durchgerechnete Beispiele legt, die der Praxis entnommen sind und verschiedene Gebiete der technischen Physik betreffen, besonders die industrielle Feinmeßtechnik, die Laboratoriumsmeßtechnik und die geodätische Vermessungstechnik. Das Buch ist vor allem für den wissenschaftlichen Nachwuchs an Hoch- und Fachschulen gedacht. Es soll aber auch zum Nachschlagen dienen und wendet sich deshalb auch an den in der Industrie und Forschung tätigen Praktiker.

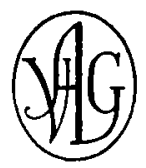

Akademische Verlagsgesellschaft Geest \& Portig K..-G., Leipzig

Die „FORTSCHRITTE DER PHYSIK“" sind durch den Buchhandel zu beziehen.

Falls keine Bezugsmöglichkeit durch eine Buchhandlung vorhanden ist, wenden Sie sich bitte in der Deutschen Demokratischen Republik

an den AKADEMIE-VERLAG, GmbH, 108 Berlin 8. Leipziger Straße 3-4

in der Deutschen Bundesrepublik

an die Auslieferungsstelle: KUNST UND WISSEN, Inhaber Erich Bieber, 7 Stuttgart, Wilhelmstraße 4-6

bei Wohnsitz im übrigen Ausland an den Deutschen Buch-Export und -Import, GmbH, 701 Leipzig 1, Postschließfach 276 oder direkt an den AKADEMIE-VERLAG, GmbH, 108 Berlin 8, Leipziger Straße 3 - 4 